\title{
Novel Hybrid FETD-FDTD Formulations for Dispersive Media
}

\author{
Ali Akbarzadeh-Sharbaf and Dennis D. Giannacopoulos
}

\begin{abstract}
Department of Electrical and Computer Engineering, McGill University, Montreal, QC H3A 0E9, Canada
\end{abstract}
\begin{abstract}
Two hybrid formulations combining the finite-element time-domain and the finite-difference time-domain methods are proposed for modeling dispersive materials. The methods are coupled to each other in a non-overlapping fashion using a new finite-element tearing and interconnecting algorithm. In addition, one of them is a fully unconditionally stable hybrid method, in which the time step is not restricted by a stability criterion. The effect of medium dispersion is considered using the Möbius transformation technique. The proposed methods are validated through numerical studies.
\end{abstract}

Index Terms-Dispersive media, finite-difference time-domain (FDTD), finite-element tearing and interconnecting (FETI), finite-element time-domain (FETD).

\section{INTRODUCTION}

$\mathbf{H}$ YBRID finite-element time-domain (FETD)-finitedifference time-domain (FDTD) is an attractive scheme, which combines the efficiency of the FDTD with the ability of the FETD in modeling complex structures. Early attempts to develop, such a hybrid method all partially encountered failure due to the occurrence of the late-time instability [1]- [3]. Finally, Rylander developed the first stable hybrid FETD-FDTD formulation [4] and proved the stability of his method [5]. The key point in this success was the equivalence between FETD and FDTD on a Cartesian grid: if the mass and stiffness matrices of the FETD are evaluated using the trapezoidal rule, which is called mass-lumping, FETD identically reduces to the FDTD formulation in space [6]. Hence, in the case of using a similar time-stepping scheme to discretize them in time, they become temporally and spatially equivalent to each other. Since the Newmark- $\beta$ (NB) method is equivalent to the central-difference method for $\beta=0$, a brick-shaped mass-lumped FETD grid with $\beta=0$ has the same update process as the standard leap-frog FDTD. The unstructured FETD part was interfaced with the FDTD by being wrapped with a single layer of such a grid. This additional layer, because of underlying equivalence, can be combined with the FDTD formulation in a stable and simple manner. Since then, all hybrid formulations have utilized the same idea [7], [12] (the only exception is a 2-D overlapping hybrid method whose stability was tested just through numerical studies [13]). Despite the contributions and advantages of this method, it has two main shortcomings: 1) there is a transition layer shared between FETD and FDTD methods and 2) only the standard leap-frog FDTD is utilized in the FDTD part, which makes the whole hybrid method conditionally stable (CS). In [9], it was attempted to mitigate the second drawback, but the method suffers from severe dissipation.

Modeling dispersive materials have gained a great deal of attention in recent years due to their widespread applications [14]. In [10], just electric and magnetic losses

Manuscript received May 25, 2014; revised July 23, 2014; accepted September 2, 2014. Date of current version April 22, 2015. Corresponding author: A. Akbarzadeh-Sharbaf (e-mail: ali.akbarzadehsharbaf@mail. mcgill.ca).

Color versions of one or more of the figures in this paper are available online at http://ieeexplore.ieee.org.

Digital Object Identifier 10.1109/TMAG.2014.2355593 were incorporated into the hybrid method. In [11] and [12], the stable hybrid FETD-FDTD method was extended to dispersive media. The former applied the recursive convolution (RC) method to the formulation based on the vector wave equation (VWE) and the latter utilized the auxiliary differential equation method for the mixed formulation in the FETD part.

The aim of this paper is to introduce a new way to couple FDTD and FETD methods in dispersive media. The FETD part is based on the VWE formulation discretized using the NB method; we name it NB-FETD. The same formulation is also utilized in the FDTD part; however, with brick-shaped mass-lumped elements, which reduces to the standard FDTD discretization in space. For the time discretization step, the value of $\beta$ is set to $1 / 4$ in the FETD region, which is known to be unconditionally stable (US) [15]; however, two different values of $\beta=0$ and $1 / 4$ are utilized in the FDTD part, resulting in two hybrid formulations. They are hybridized with each other with a new finite-element tearing and interconnecting (FETI) algorithm. Hence, there is no need to employ any transition region and they are non-overlapping. In addition, the formulation is extended to dispersive media using the Möbius transformation approach [16], [17], which is simpler and more flexible than the RC-based methods previously employed and can be considered as another contribution of this paper.

\section{Formulation}

\section{A. FETD Formulations and Equivalences to the FDTD}

There are two main FETD formulations, one is based on the VWE

$$
[\mathcal{S}]\{e\}+[\mathcal{M}] \frac{\partial^{2}\{e\}}{\partial t^{2}}=\{f\}
$$

while the other involves directly solving the Maxwell's equations

$$
\begin{aligned}
{[\mathcal{M}] \frac{\partial\{e\}}{\partial t} } & =[\mathcal{C}]^{T}\left[\mathcal{M}_{f}\right]\{b\} \\
\frac{\partial\{b\}}{\partial t} & =-[\mathcal{C}]\{e\}
\end{aligned}
$$

where $\mathcal{M}_{i j}=\int_{\Omega} \varepsilon \boldsymbol{W}_{i}^{(1)} \cdot \boldsymbol{W}_{j}^{(1)} d V, \mathcal{S}_{i j}=\int_{\Omega} \mu^{-1} \nabla \times \boldsymbol{W}_{i}^{(1)}$. $\nabla \times \boldsymbol{W}_{j}^{(1)} d V, \mathcal{M}_{f_{i j}}=\int_{\Omega} \mu^{-1} \boldsymbol{W}_{i}^{(2)} \cdot \boldsymbol{W}_{j}^{(2)} d V, f_{i}=$ $-\int_{\Omega} \boldsymbol{W}_{i}^{(1)} \cdot \partial \mathcal{J}_{\text {imp }}(t) / \partial t d V$, and $[\mathcal{C}]$ is the incidence matrix. Eliminating $\{b\}$ in (2) leads to the VWE-based formulation (1), in which $[\mathcal{S}]$ can be shown to be equal to $[\mathcal{C}]^{T}\left[\mathcal{M}_{f}\right][\mathcal{C}]$ thanks 
to the properties of Whitney 1-form, $\boldsymbol{W}^{(1)}$, and 2-form, $\boldsymbol{W}^{(2)}$, elements. Hence, both formulations become equivalent to each other in the continuous-time case.

On the other hand, it can be shown that (2) can exactly reduce to the standard FDTD equations in space, if the $[\mathcal{M}]$ and $\left[\mathcal{M}_{f}\right]$ matrices are approximated using the trapezoidal integration rule over rectangular elements in 2-D or brickshaped elements in 3-D [6]. In the case of mass-lumping, there still exists the same equivalence between two FETD formulations, if the $[\mathcal{S}]$ matrix is also calculated using the trapezoidal integration. Hence, (1) and (2) and the standard FDTD methods are all spatially equivalent to each other on mass-lumped Cartesian grids. Hence, we can conclude that the fully discretized form of all three mentioned formulations are equivalent to each other, if the same timediscretization method is employed for all of them. Here, two more widely-used methods are considered: 1) the centraldifference and 2) the trapezoidal schemes. These methods can be easily applied to the semidiscrete mixed FETD formulation (2), which results in the leap-frog FETD [18] and the Crank-Nicolson (CN)-FETD methods [19], respectively. Since the semidiscrete FDTD equations have exactly the same form as the mixed FETD (2), application of the above two methods results in a similar result: 1$)$ the standard leap-frog FDTD and 2) the CN-FDTD [20]. It should be noted that the CN-FDTD has been proven to be US [20].

The NB method, which has been widely used to discretize the VWE-based FETD (1), consists of the following approximations [21]:

$$
\begin{aligned}
& \beta \frac{\partial\{e\}}{\partial t}^{n+1}+(1-2 \beta){\frac{\partial\{e\}^{n}}{\partial t}}^{n}+\beta \frac{\partial\{e\}}{\partial t}^{n-1} \\
& =\frac{\{e\}^{n+1}-\{e\}^{n-1}}{2 \Delta t}
\end{aligned}
$$

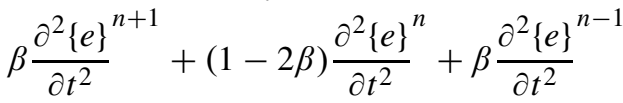

$$
\begin{aligned}
& =\frac{\{e\}^{n+1}-2\{e\}^{n}+\{e\}^{n-1}}{\Delta t^{2}} .
\end{aligned}
$$

It is clear that choosing $\beta=0$ and $1 / 4$ is equivalent to discretizing the first and second time derivatives using the central-difference and trapezoidal rule, respectively. Hence, $\beta=0$ and $1 / 4$ make the VWE FETD formulation (1) temporally equivalent to the leap-frog mixed FETD and the CN-FETD formulations, respectively. All in all, the VWE FETD formulation with mass-lumped matrices on a Cartesian grid and temporally discretized with the NB method with $\beta=$ 0 and $1 / 4$ is equivalent to the leap-frog FDTD and CN-FDTD formulations. The first equivalence has been long-known and utilized in the development of the stable hybrid method [4], however, the second one has been recently revealed [17].

In this paper, although there are several possibilities, we mainly focus on two hybrid methods. The NB-FETD with $\beta=1 / 4$ is utilized in the unstructured part of both hybrid formulations, however, $\beta=0$ and $1 / 4$ are employed in the FDTD part of each hybrid method. We call them CS-hybrid and US-hybrid methods, respectively. The first one is equivalent to combining the standard leap-frog FDTD with the US NB-FETD and the second one is equivalent to combining the CN-FDTD with the US NB-FETD. Our numerical studies show that the hybrid method remains stable, if the time step value satisfies the stability condition of each region; hence, the US-hybrid method is stable for arbitrary large time steps.

\section{B. Coupling Using the FETI}

The FETI tears the computational domain into several (here two FETD and FDTD) non-overlapping sub-domains and solves the VWE in each sub-domain separately. Then, it interconnects sub-domains together by solving an interface problem, which enforces continuity of a quantity along interface. The FETIs in electromagnetics are usually based on enforcing continuity of the tangential component of the electric field $e(t)$ [22], [23], however, we enforce continuity of $\partial e(t) / \partial t$. It can be shown that this interface condition does not add or remove energy, hence, it preserves stability of the problem in each sub-domain [24]. It should be noted that continuity of $e(t)$ implies continuity of $\partial e(t) / \partial t$ in the continuous-time case, but not in the discrete-time case and each results in different properties.

Consider, the VWE FETD (1) in each region at $t=n \Delta t$

$$
\left[\mathcal{S}_{i}\right]\left\{e_{i}\right\}^{n}+\left[\mathcal{M}_{i}\right] \frac{\partial^{2}\left\{e_{i}\right\}^{n}}{\partial t^{2}}=\left\{f_{i}\right\}^{n}
$$

the continuity condition between sub-domains can be expressed as the following constraint:

$$
\sum_{i}\left[B_{i}\right]^{T}{\frac{\partial\left\{e_{i}\right\}^{n}}{\partial t}}^{n}=\{0\}
$$

where $\left[B_{i}\right]$ is a Boolean matrix that picks out the interface degrees of freedom (DOFs). Solving (4) in each region together with the constraint (5) is equivalent to solving the following modified equation:

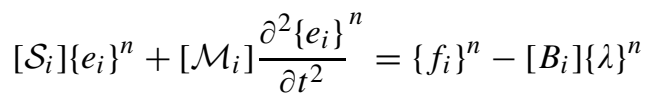

which can be discretized using the NB method [21]. The $\{\lambda\}^{n}$ can be calculated using

$$
\left.\begin{array}{l}
\left(\sum_{i}\left[B_{i}\right]^{T}\left[\mathcal{Q}_{i}\right]^{-1}\left[B_{i}\right]\right)\{\lambda\}^{n} \\
=\sum_{i}\left\{\left[B_{i}\right]^{T}\left[\mathcal{Q}_{i}\right]^{-1}\left\{f_{i}\right\}^{n-1}-\left[B_{i}\right]^{T}\left[\mathcal{Q}_{i}\right]^{-1}\left[\mathcal{S}_{i}\right]\left\{e_{i}\right\}^{n-1}\right. \\
\quad+\left(\frac{2}{\Delta t}\left[B_{i}\right]^{T}-\Delta t\left[B_{i}\right]^{T}\left[\mathcal{Q}_{i}\right]^{-1}\left[\mathcal{S}_{i}\right]\right) \frac{\partial\left\{e_{i}\right\}^{n-1}}{\partial t} \\
\quad+\left(\left[B_{i}\right]^{T}+\Delta t^{2}\left(\beta_{i}-0.5\right)\left[B_{i}\right]^{T}\left[\mathcal{Q}_{i}\right]^{-1}\left[\mathcal{S}_{i}\right]\right) \frac{\partial^{2}\left\{e_{i}\right\}^{n-1}}{\partial t^{2}}
\end{array}\right\}
$$

where $\left[\mathcal{Q}_{i}\right]=\left[\mathcal{M}_{i}\right]+\beta_{i} \Delta t^{2}\left[\mathcal{S}_{i}\right]$. It should be noted that the size of (7) is equal to the number of interface DOFs.

\section{Medium Dispersion}

Without loss of generality, imagine each sub-domain is homogeneously filled with a dispersive material with the 


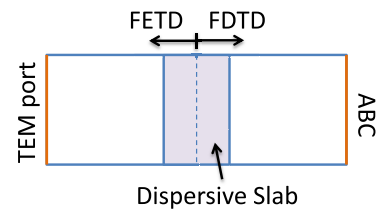

Fig. 1. 2-D view of the problem. 2-cm-wide dispersive dielectric slab is placed in the middle of a 3-D parallel-plate waveguide.

following permittivity and permeability:

$$
\varepsilon_{i}(s)=\frac{\sum_{n=0}^{p_{i}} a_{n_{i}} s^{n}}{\sum_{n=0}^{p_{i}} b_{n_{i}} s^{n}}, \quad \mu_{i}(s)=\frac{\sum_{n=0}^{p_{i}} \alpha_{n_{i}} s^{n}}{\sum_{n=0}^{p_{i}} \beta_{n_{i}} s^{n}} .
$$

Using the Möbius transformation technique described in [16] and [17], (8) can be directly discretized as

$$
\begin{aligned}
\varepsilon_{i}(z) & =\frac{c_{0_{i}}+c_{1_{i}} z^{-1}+\cdots+c_{\left(p_{i}\right)_{i}} z^{-p_{i}}}{1+d_{1_{i}} z^{-1}+\cdots+d_{\left(p_{i}\right)_{i}} z^{-p_{i}}} \\
\mu_{i}^{-1}(z) & =\frac{q_{0_{i}}+q_{1_{i}} z^{-1}+\cdots+q_{\left(p_{i}\right)_{i}} z^{-p_{i}}}{1+r_{1_{i}} z^{-1}+\cdots+r_{\left(p_{i}\right)_{i}} z^{-p_{i}}}
\end{aligned}
$$

by replacing $s$ with $2 / \Delta t 1-z^{-1} / 1+z^{-1}$ in (8). Since the medium is homogeneous, the permittivity and permeability in (6) can be pulled out of the integral and expressed as $\left\{\mathcal{L}_{\varepsilon_{i}}\right\}^{n}=\varepsilon_{i}(z)\left[\mathcal{M}_{i}\right] \partial^{2}\left\{e_{i}\right\} / \partial t^{2^{n}}$ and $\left\{\mathcal{L}_{\mu_{i}^{-1}}\right\}^{n}=\mu_{i}^{-1}(z)\left[\mathcal{S}_{i}\right]\left\{e_{i}\right\}^{n}$. Where $\left\{\mathcal{L}_{\varepsilon_{i}}\right\}$ and $\left\{\mathcal{L}_{\mu_{i}^{-1}}\right\}$ are auxiliary variables and can be updated as [17]

$$
\begin{aligned}
\left\{\mathcal{L}_{\varepsilon_{i}}\right\}^{n} & =c_{0_{i}}\left[\mathcal{M}_{i}\right] \frac{\partial^{2}\left\{e_{i}\right\}^{n}}{\partial t^{2}}+\left\{\mathcal{W}_{1, i}\right\}^{n-1} \\
\left\{\mathcal{L}_{\mu_{i}^{-1}}\right\}^{n} & =q_{0_{i}}\left[\mathcal{S}_{i}\right]\left\{e_{i}\right\}^{n}+\left\{\mathcal{G}_{1, i}\right\}^{n-1}
\end{aligned}
$$

where $\left\{\mathcal{W}_{1, i}\right\}$ and $\left\{\mathcal{G}_{1, i}\right\}$ are auxiliary variables that have been employed to make the implementation memory-efficient and can be updated as

$$
\begin{gathered}
\left\{\mathcal{W}_{\alpha, i}\right\}^{n}=c_{\alpha_{i}}\left[\mathcal{M}_{i}\right]{\frac{\partial^{2}\left\{e_{i}\right\}}{\partial t^{2}}}^{n}-d_{\alpha_{i}}\left\{\mathcal{L}_{\varepsilon_{i}}\right\}^{n}+\left\{\mathcal{W}_{\alpha+1, i}\right\}^{n-1} \\
\alpha=1,2, \cdots, p_{i}-1 \\
\left\{\mathcal{W}_{\alpha, i}\right\}^{n}=c_{\alpha_{i}}\left[\mathcal{M}_{i}\right]{\frac{\partial^{2}\left\{e_{i}\right\}}{\partial t^{2}}}^{n}-d_{\alpha_{i}}\left\{\mathcal{L}_{\varepsilon_{i}}\right\}^{n} ; \quad \alpha=p_{i} \\
\left\{\mathcal{G}_{\alpha, i}\right\}^{n}=q_{\alpha_{i}}\left[\mathcal{S}_{i}\right]\left\{e_{i}\right\}^{n}-r_{\alpha_{i}}\left\{\mathcal{L}_{\mu_{i}^{-1}}\right\}^{n}+\left\{\mathcal{G}_{\alpha+1, i}\right\}^{n-1} \\
\alpha=1,2, \cdots, p_{i}-1 \\
\left\{\mathcal{G}_{\alpha, i}\right\}^{n}=q_{\alpha_{i}}\left[\mathcal{S}_{i}\right]\left\{e_{i}\right\}^{n}-r_{\alpha_{i}}\left\{\mathcal{L}_{\mu_{i}^{-1}}\right\}^{n} ; \quad \alpha=p_{i}
\end{gathered}
$$

substituting (11) and (12) in (6), gives

$$
q_{0_{i}}\left[\mathcal{S}_{i}\right]\left\{e_{i}\right\}^{n}+c_{0_{i}}\left[\mathcal{M}_{i}\right]{\frac{\partial^{2}\left\{e_{i}\right\}^{n}}{\partial t^{2}}}^{n}=\left\{g_{i}\right\}^{n}-\left[B_{i}\right]\{\lambda\}^{n}
$$

where

$$
\left\{g_{i}\right\}^{n}=\left\{f_{i}\right\}^{n}-\left\{\mathcal{W}_{1, i}\right\}^{n-1}-\left\{\mathcal{G}_{1, i}\right\}^{n-1}
$$

comparing (15) with (6), $\{\lambda\}$ (7) has to be modified when the interface of the hybrid method is placed in a dispersive media.

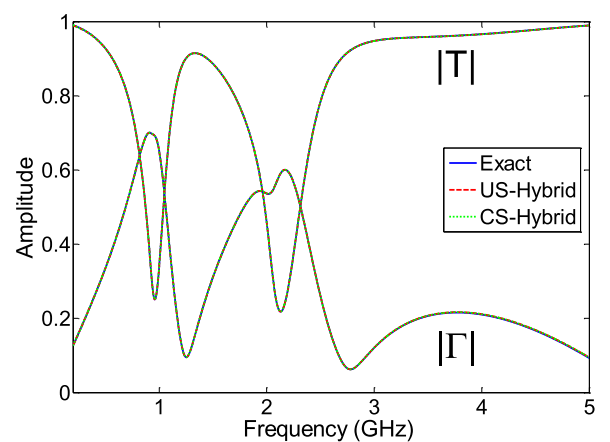

Fig. 2. Numerical results along with the exact solutions for the electrically dispersive dielectric slab.

\section{NumericAl RESUlts}

To validate the formulations described earlier, we consider a 3-D numerical example whose analytical solution is available. As shown in Fig. 1, a $2 \mathrm{~cm}$ wide dispersive dielectric slab is placed in the middle of a parallel-plate waveguide. One side of the problem, including half of the slab, is solved with the FETD and the other half with the FDTD method. The transition between tetrahedral elements of the FETD part and brick-shaped elements of the FDTD part is made by pyramidal elements. One side of the waveguide is excited by the TEM mode with the differentiated Blackman-Harris pulse shape with $f_{\mathrm{bw}}=1.55 / T=5 \mathrm{GHz}$ and the other side is terminated by a first-order absorbing boundary condition. Both CS-hybrid and US-hybrid methods are employed. To have a stable solution, the time step is set to $95 \%$ of the Courant-Friedrichs-Lewy (CFL) condition of the leap-frog FDTD method for the CS-hybrid method $\left(\Delta t_{\mathrm{CS}}=0.95 \Delta t_{\mathrm{CFL}}\right)$, while the size of the time step for the US-hybrid is double ( $\left.\Delta t_{\mathrm{US}}=2 \Delta t_{\mathrm{CS}}\right)$. The dispersion of the slab is characterized with different material models.

In the first example, an electrically dispersive model is considered $\left(\mu=\mu_{0}\right)$. Permittivity of the slab is characterized with a fourth-order, including two Lorentz pole-pairs, model as follows:

$$
\varepsilon(s)=\varepsilon_{\infty}+G_{e_{1}} \frac{\left(\varepsilon_{s}-\varepsilon_{\infty}\right) \omega_{e_{1}}^{2}}{s^{2}+2 \delta_{e_{1}} s+\omega_{e_{1}}^{2}}+G_{e_{2}} \frac{\left(\varepsilon_{s}-\varepsilon_{\infty}\right) \omega_{e_{2}}^{2}}{s^{2}+2 \delta_{e_{2}} s+\omega_{e_{2}}^{2}}
$$

where the parameters are given as

$$
\begin{aligned}
G_{e_{1}} & =0.7, G_{e_{2}}=0.3, \delta_{e_{1}}=0.05 \omega_{e_{1}}, \delta_{e_{2}}=0.05 \omega_{e_{2}} \\
\varepsilon_{s} & =4 \varepsilon_{0}, \varepsilon_{\infty}=2 \varepsilon_{0}, \omega_{e_{1}}=1.9 \pi \times 10^{9}, \omega_{e_{2}}=4.2 \pi \times 10^{9}
\end{aligned}
$$

the magnitude of the reflection $(\Gamma)$ and transmission $(T)$ coefficients of the dielectric slab obtained by both hybrid methods along with the exact solutions are shown in Fig. 2.

As the next example, a magnetically dispersive slab with a model similar to (17) is considered. The permittivity of the slab is $\varepsilon_{0}$ and the permeability model has the following parameters:

$$
\begin{aligned}
G_{m_{1}} & =0.4, G_{m_{1}}=0.6, \delta_{m_{1}}=0.03 \omega_{m_{1}} \\
\delta_{m_{2}} & =0.025 \omega_{m_{2}}, \mu_{s}=5.5 \mu_{0}, \mu_{\infty}=3.4 \mu_{0} \\
\omega_{m_{1}} & =1.5 \pi \times 10^{9}, \omega_{m_{2}}=4.5 \pi \times 10^{9} .
\end{aligned}
$$

Fig. 3 shows the results pertaining to this case. 


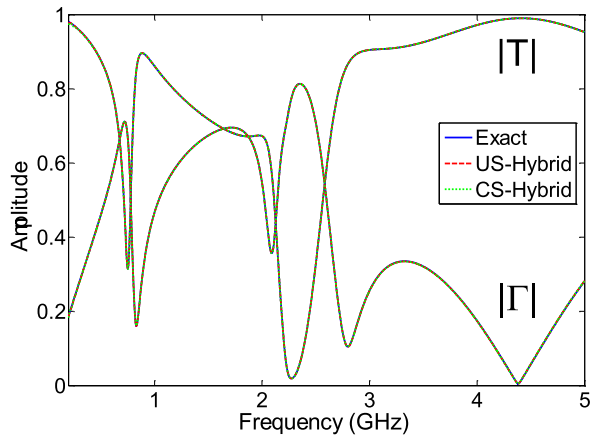

Fig. 3. Numerical results along with the exact solutions for the magnetically dispersive dielectric slab.

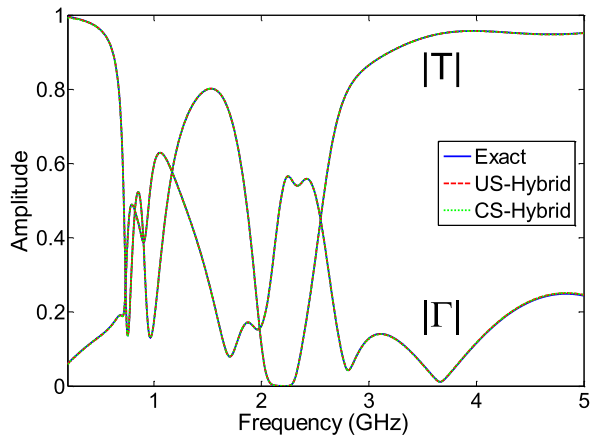

Fig. 4. Numerical results along with the exact solutions for the doubly dispersive dielectric slab.

Finally, a doubly dispersive slab, combining electric and magnetic dispersion behaviors of the previous examples, is employed. The reflection and transmission coefficients are shown in Fig. 4.

As can be seen, in all cases the US-hybrid and CS-hybrid results match with the exact solutions very well. In addition, all examples were simulated up to several hundred thousand time steps and no sign of instability, late-time growth, or dissipation were observed. Furthermore, the US-hybrid method was tested with very large time steps, but it always remained stable.

\section{CONCLUSION}

A novel and general approach to combine the FETD and FDTD formulations in dispersive media has been proposed. It has two distinct advantages over the previous methods: 1) it is a non-overlapping method and 2) it can be stable for arbitrary large time steps. The Möbius transformation technique has been utilized to include dispersive media, which has made the formulation more flexible and easier-to-implement than the conventional RC methods utilized in the VWE-based formulations.

\section{REFERENCES}

[1] R.-B. Wu and T. Itoh, "Hybrid finite-difference time-domain modeling of curved surfaces using tetrahedral edge elements," IEEE Trans. Antennas Propag., vol. 45, no. 8, pp. 1302-1309, Aug. 1997.

[2] D. Riley and C. D. Turner, "Interfacing unstructured tetrahedron grids to structured-grid FDTD," IEEE Microw. Guided Wave Lett., vol. 5, no. 9, pp. 284-286, Sep. 1995.
[3] A. Monorchio and R. Mittra, "Time-domain (FE/FDTD) technique for solving complex electromagnetic problems," IEEE Microw. Guided Wave Lett., vol. 8, no. 2, pp. 93-95, Feb. 1998.

[4] T. Rylander and A. Bondeson, "Stable FEM-FDTD hybrid method for Maxwell's equations," Comput. Phys. Commun., vol. 125, nos. 1-3, pp. 75-82, Mar. 2000.

[5] T. Rylander and A. Bondeson, "Stability of explicit-implicit hybrid time-stepping schemes for Maxwell's equations," J. Comput. Phys., vol. 179, no. 2, pp. 426-438, Jul. 2002.

[6] G. Cohen and P. Monk, "Gauss point mass lumping schemes for Maxwell's equations," Numer. Methods Partial Differ. Equ., vol. 14, no. 1 , pp. 63-88, 1998.

[7] D. Degerfeldt and T. Rylander, "A brick-tetrahedron finite-element interface with stable hybrid explicit-implicit time-stepping for Maxwell's equations," J. Comput. Phys., vol. 220, no. 1, pp. 383-393, Dec. 2006.

[8] B. Zhu, J. Chen, W. Zhong, and Q. H. Liu, "A hybrid FETD-FDTD method with nonconforming meshes," Commun. Comput. Phys., vol. 9, no. 3, pp. 828-842, Mar. 2011.

[9] A. A. Sharbaf and R. Sarraf-Shirazi, "An unconditionally stable hybrid FETD-FDTD formulation based on the alternating-direction implicit algorithm," IEEE Antennas Wireless Propag. Lett., vol. 9 , pp. 1174-1177, 2010.

[10] T. Halleröd and T. Rylander, "Electric and magnetic losses modeled by a stable hybrid with explicit-implicit time-stepping for Maxwell's equations," J. Comput. Phys., vol. 227, no. 9, pp. 4499-4511, Apr. 2008.

[11] F. Edelvik and B. Strand, "Frequency dispersive materials for 3-D hybrid solvers in time domain," IEEE Trans. Antennas Propag., vol. 51, no. 6 , pp. 1199-1205, Jun. 2003.

[12] B. Donderici, "Time-domain solvers for complex-media electrodynamics and plasma physics," Ph.D. dissertation, Dept. Elect. Comput. Eng., Ohio State Univ., Columbus, OH, USA, 2008.

[13] E. Abenius, U. Andersson, F. Edelvik, L. Eriksson, and G. Ledfelt, "Hybrid time domain solvers for the Maxwell equations in 2D," Int. J. Numer. Methods Eng., vol. 53, no. 9, pp. 2185-2199, 2002.

[14] F. L. Teixeira, "Time-domain finite-difference and finite-element methods for Maxwell equations in complex media," IEEE Trans. Antennas Propag., vol. 56, no. 8, pp. 2150-2166, Aug. 2008.

[15] S. D. Gedney and U. Navsariwala, "An unconditionally stable finite element time-domain solution of the vector wave equation," IEEE Microw. Guided Wave Lett., vol. 5, no. 10, pp. 332-334, Oct. 1995.

[16] J. A. Pereda, A. Vegas, and A. Prieto, "FDTD modeling of wave propagation in dispersive media by using the Möbius transformation technique," IEEE Trans. Microw. Theory Techn., vol. 50, no. 7, pp. 1689-1695, Jul. 2002.

[17] A. Akbarzadeh-Sharbaf and D. D. Giannacopoulos, "Finite-element time-domain solution of the vector wave equation in doubly dispersive media using Möbius transformation technique," IEEE Trans. Antennas Propag., vol. 61, no. 8, pp. 4158-4166, Aug. 2013.

[18] M. F. Wong, O. Picon, and V. F. Hanna, "A finite element method based on Whitney forms to solve Maxwell equations in the time domain," IEEE Trans. Magn., vol. 31, no. 3, pp. 1618-1621, May 1995.

[19] M. Movahhedi, A. Abdipour, A. Nentchev, M. Dehghan, and S. Selberherr, "Alternating-direction implicit formulation of the finiteelement time-domain method," IEEE Trans. Microw. Theory Techn., vol. 55, no. 6, pp. 1322-1331, Jun. 2007.

[20] G. Sun and C. W. Trueman, "Unconditionally-stable FDTD method based on Crank-Nicolson scheme for solving three-dimensional Maxwell equations," Electron. Lett., vol. 40, no. 10, pp. 589-590, May 2004.

[21] N. M. Newmark, "A method of computation for structural dynamics," J. Eng. Mech. Division, vol. 85, no. 3, pp. 67-94, Jul. 1959.

[22] U. D. Navsariwala and S. D. Gedney, "An efficient implementation of the finite-element time-domain algorithm on parallel computers using a finite-element tearing and interconnecting algorithm," Microw. Opt. Technol. Lett., vol. 16, no. 4, pp. 204-208, Nov. 1997.

[23] L. Du, R. S. Chen, Z. B. Ye, and Y. Yang, "An efficient time-domain method analysis of quasiperiodic structures by a finite-element tearing and interconnecting algorithm," Microw. Opt. Technol. Lett., vol. 52, no. 5, pp. 1072-1078, May 2010.

[24] A. Gravouil and A. Combescure, "Multi-time-step explicit-implicit method for non-linear structural dynamics," Int. J. Numer. Methods Eng., vol. 50, no. 1, pp. 199-225, Jan. 2001. 